\title{
ORIGINAL ARTICLE \\ Early low-frequency stimulation of the pudendal nerve can inhibit detrusor overactivity and delay progress of bladder fibrosis in dogs with spinal cord injuries
}

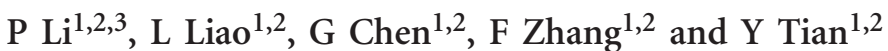

Objectives: To determine the inhibitory effects of pudendal nerve stimulation $(5 \mathrm{~Hz})$ on bladder overactivity at the early stage of spinal cord injury (SCl) in dogs, and to explore the possible effects on delayed progression of bladder fibrosis after SCl.

Methods: The study was performed using six dogs with spinal cord transection at the T9-T10 level. Group 1 (three dogs) underwent low-frequency electrical stimulation of the pudendal nerve 1 day after spinal cord transection. Group 2 (three dogs) underwent only spinal cord transection. All dogs underwent urodynamic examination at 1 and 3 months after SCl. The bladders were removed for histological examination of fibrosis at 3 months after SCl.

Results: Bladder capacity and compliance were significantly increased $(P<0.05)$ by pudendal nerve stimulation in group 1 when compared with group 2 at 1 and 3 months after SCI. Non-voiding contractions (NVCs) were inhibited in group 1 compared with group 2. Collagen fibers were significantly increased and elastic fibers were significantly decreased $(P<0.05)$ in group 2 when compared with group 1.

Conclusion: Early low-frequency pudendal nerve stimulation can inhibit detrusor overactivity (DO), increase bladder capacity and delay the progression of bladder fibrosis.

Spinal Cord (2013) 51, 668-672; doi:10.1038/sc.2013.60; published online 25 June 2013

Keywords: bladder; dogs; pudendal nerve stimulation; spinal cord injury; urodynamics

\section{INTRODUCTION}

Neurogenic bladder dysfunction after spinal cord injury (SCI) is a major medical and social problem. SCI between the brain-stem and the lumbosacral spinal cord results in dysfunction of the lower urinary tract, including neurogenic detrusor overactivity (NDO) and detrusor sphincter dyssynergia. ${ }^{1}$ Patients with SCI who have NDO and/or detrusor sphincter dyssynergia often have transient high intravesical pressures, low maximum cystometric capacity (MCC), low bladder compliance (BC), large residual urine volumes and incontinence. Recurrent urinary tract infections and vesicoureteral reflux are common after $\mathrm{SCI}$, which can lead to upper urinary tract damage, and ultimately to renal failure. ${ }^{2}$ The main reason for high intravesical pressures, low BC and vesicoureteral reflux is bladder fibrosis. As such, prevention of bladder fibrosis may be an effective method to protect the bladder and upper urinary tract function in these patients. Up to now, however, there is no effective method for preventing bladder fibrosis.

Currently, clean intermittent catheterization combined with anticholinergic medication is the gold-standard treatment for $\mathrm{NDO}^{3}$ However, there are several limitations to this treatment, including drug-related side effects, urethral damage and recurrent urinary tract infections, which can significantly diminish the patient's quality of life. ${ }^{4}$

Electrical stimulation has been introduced as another treatment option for urinary incontinence caused by NDO. Afferent pudendal nerve electrical stimulation (PNS) has been demonstrated to inhibit the micturition reflex, abolish uninhibited bladder contractions and increase MCC in animals and humans with SCI. ${ }^{5,6}$ These results indicate that PNS might be a promising treatment option for neurogenic bladder overactivity after SCI.

Previous PNS studies have shown immediate suppressive effects on NDO during electrical stimulation in patients with chronic $\mathrm{SCI},{ }^{6,7}$ but there is no report regarding the inhibitory effect of PNS on bladder overactivity at the early stage of SCI, or concerning the effects of early PNS on prevention or delay of bladder fibrosis. Our prior study shows that PNS can inhibit non-voiding contraction (NVC) during bladder filling in dogs with chronic SCI, but this can only increase MCC at an early stage (1 month), and the histological results indicate that bladder fibrosis may be the main reason for the absence of an effect during the chronic period (6 months). ${ }^{8}$ As such, it is questionable if early electrical stimulation of the pudendal nerve is appropriate for patients with SCI.

The present study aims to determine the inhibitory effects of pudendal nerve stimulation $(5 \mathrm{~Hz})$ on bladder overactivity at the early stage of SCI in dogs, and to explore its possible effects on delaying the progress of bladder fibrosis.

\section{MATERIALS AND METHODS}

\section{Animal care}

All animal care and experimental procedures were followed according to the National Institutes of Health guidelines, and were approved by the Institutional Animal Care and Use Committee of Capital Medical University, China. 


\section{SCI and electrode implantation}

Spinal cord transection and electrode implantation were performed in six adult, male beagle dogs $(10.0-13.0 \mathrm{~kg})$ at the T9-T10 vertebral level via dorsal laminectomy under pentobarbital sodium $\left(30-35 \mathrm{mg} \mathrm{kg}^{-1}\right.$ ) anesthesia and aseptic conditions. After the spinal cord was completely cut, a piece of gel foam was placed between the cut ends (usually a separation of $2-3 \mathrm{~mm}$ ). The muscle and skin were then sutured. The left-side pudendal nerve was accessed posteriorly between the sciatic notch and the tail. A tripolar cuff electrode with leads made of stainless steel wire (diameter of $0.25 \mathrm{~mm}$; Micro Probe, Gaithersburg, MD, USA) and separated at a $2-\mathrm{mm}$ distance was placed around the pudendal nerve at a location central to the deep perineal branch. After implanting the electrode, a tunneling tool was used to create a tunnel at the subcutaneous level from the electrode to the dorsal skin. Removal of the tunneling tool resulted in the tube being left in place in the tunnel through which the leads were fed. Subsequently, the tube was removed, leaving the leads in position. The muscle and skin were then sutured. After a full recovery from anesthesia, the animals were returned to their cages. Antibiotics (amoxicillin trihydrate/clavulanate potassium, $15-20 \mathrm{mg} \mathrm{kg}^{-1}$ ) were administered at the time of surgery and again the next day. The bladders were manually intermittent catheterized twice a day to prevent overdistension and infection after surgery, and continued throughout the 3-month study.

\section{Pudendal nerve stimulation}

The six dogs were randomly allocated into two groups. Group 1 (three dogs) underwent electrical stimulation of the pudendal nerve 1 day after transection (early stage), with the stimulation applied 5 days a week ( $30 \mathrm{~min}$ each day) for 3 months. Biphasic pulses (pulse width of $0.2 \mathrm{msec}$ ) of 1 - to $10-\mathrm{V}$ intensity were used to stimulate the pudendal nerve at a frequency of $5 \mathrm{~Hz}$. The stimulation intensity was determined at the beginning of its effectiveness to induce anal sphincter contraction at $5 \mathrm{~Hz}$ each day. A test stimulator (Interstim Model 3625; Medtronic, Minneapolis, MN, USA) was used to generate the stimulus pulses. Group 2 (three dogs) did not undergo electrical stimulation of the pudendal nerve.

\section{Urodynamic analysis}

All dogs underwent standard water cystometry in the supine position three times: before spinal cord transection as well as at 1 and 3 months after transection. The animals were anesthetized with a-chloralose $\left(65 \mathrm{mg} \mathrm{kg}^{-1}\right.$, intravenously, supplemented at $15 \mathrm{mg} \mathrm{kg}^{-1}$; Sigma-Aldrich, St Louis, MO, USA). A double lumen, 5 French, catheter was inserted into the bladder via the urethra and secured by a ligature. One lumen of the catheter was attached to a pump to infuse the bladder with saline, and the other lumen was connected to a urodynamic analyzer (Andromeda, Medizinische Systeme GmbH, Taufkirchen, Germany). All cystometrograms were preformed without stimulation of the pudendal nerve at least for $12 \mathrm{~h}$. Slow infusion $\left(10 \mathrm{ml} \mathrm{min}^{-1}\right)$ of the bladder was always initiated with the bladder empty (that is, one-cystometrogram). When fluid was released from the bladder, the infusion was stopped immediately and the bladder was emptied. The procedure was repeated 3-6 times for each animal, with a 5-10-min interval to allow the distended detrusor to recover.

The urodynamic variables were defined according to the standards recommended by the International Continence Society. ${ }^{9,10}$ Special attention was given to the following parameters during cystometrogram: (1) MCCs defined as the infused volume at which a bladder contraction was induced and fluid was released from the bladder, or when fluid leaked from the bladder in the absence of bladder contraction; (2) BC defined as the relationship between the change in bladder volume and the change in detrusor pressure (calculated by dividing the volume change by the change in detrusor pressure) and (3) the number of NVCs defined as a bladder contraction without voiding.

\section{Histological evaluation}

The dogs were euthanized by overdose of pentobarbital sodium $\left(200 \mathrm{mg} \mathrm{kg}^{-1}\right)$ immediately after the last urodynamic study, and the bladder was removed. Specimens acquired from the anterior wall of the bladders were fixed with 10\% neutral formalin for $48-78 \mathrm{~h}$. Following fixation, the samples were embedded in paraffin for serial sectioning. Sections were stained with Ponceau S/Victoria blue. Tissues were examined with a $\mathrm{B} \times 40$ light microscope (Olympus, Tokyo, Japan). Following Ponceau S/Victoria blue staining, collagen fiber appeared red, elastic fiber appeared blue, whereas smooth muscle and red blood cells appeared yellow.

Morphometric measurements were performed with image analysis software (Image-Pro Plus 6.0; Media Cybernetics, Bethesda, MD, USA). The images of 10 randomly selected fields in Ponceau S/Victoria blue $(\times 40)$-stained sections were transferred to a computer. The proportion of the area that collagen and elastic fibers occupied was then determined.

\section{Statistical analysis}

Statistical analysis was performed using SPSS software (version 13.0 for Windows; SPSS, Chicago, IL, USA). Parameters measured from multiple trials in the same animal were averaged. To reduce the individual variation of MCC and compliance, the values were normalized to baseline prior to the experiment. The proportion of the area that collagen and elastic fibers occupied was also averaged. An independent $t$-test was used to determine the statistical significance between the two groups. $P<0.05$ was considered statistically significant.

\section{RESULTS}

\section{Functional findings}

All dogs underwent standard water cystometry without stimulation of the pudendal nerve. From the cystometrogram (Figure 1), there was detrusor overactivity (DO) in group 2 (no PNS) at 1 and 3 months post SCI, and bladder capacity and compliance were significantly decreased (Figure 1a). In group 1 (early PNS), the DO was inhibited, and bladder capacity and compliance were not decreased at 1 and 3 months post SCI (Figure 1b). The number of NVCs increased from $2.00 \pm 0.89$ in group 1 to $4.50 \pm 1.05$ in group 2 at 1 month, and from $0.83 \pm 0.75$ to $5.17 \pm 1.17$ at 3 months. Bladder capacity and
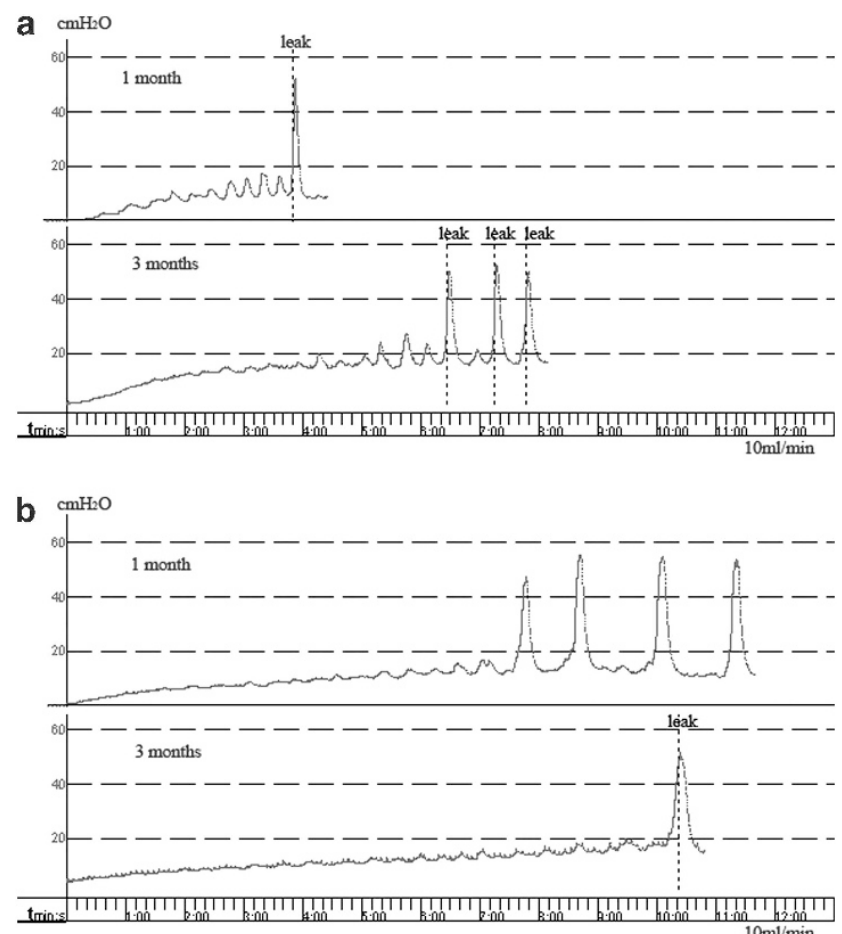

Figure 1 In group 2 (a, no PNS), DO was not inhibited, and bladder capacity and compliance were significantly decreased at 1 and 3 months post SCl. In group 1 (b, early PNS), DO was inhibited, and bladder capacity and compliance were not decreased. PNS, pudendal nerve stimulation. 
compliance were significantly increased $(P<0.05)$ in group 1 when compared with group 2 at 1 and 3 months. All capacity and compliance data are listed in Table 1 . The summary of the changes in capacity and compliance for each group is shown in Figure 2.

\section{Histological findings}

There were significant differences in the histological findings between groups 1 and 2 (Figures 3 and 4). The proportion of collagen fibers increased from $3.53 \pm 1.65 \%$ in group 1 to $6.05 \pm 2.03 \%$ in group 2, whereas the proportion of elastic fibers decreased from $1.70 \pm 0.92 \%$ in group 1 to $0.96 \pm 0.29 \%$ in group 2 (see Table 2 ).

\section{DISCUSSION}

The International Continence Society has defined NDO as an urodynamic observation, characterized by involuntary detrusor contractions during the filling phase that may be spontaneous or provoked when there is a relevant neurological condition. ${ }^{9}$ When control function in the cerebrum and the pons is blocked due to injury of the spinal cord above the sacral cord, the bladder becomes overactive, which can result in elevated bladder pressure during micturition and lead to structural damage of the bladder, vesicoureteral reflux and renal insufficiency. ${ }^{11}$ The purpose of neurogenic bladder management in patients with SCI is to preserve renal function and maintain urinary continence, consequently improving routine life and social activity. Successful management strategies should meet three main objectives: low-pressure urine storage, low-pressure voiding and adequate urine drainage.
Clean intermittent catheterization with concomitant anticholinergic medication is currently the most effective means of bladder management for most individuals with SCI. However, many patients are refractory to the medication or have dose-limiting side effects. Another treatment method is botulinum toxin injection to the detrusor muscle, but requires repeated injection. ${ }^{12}$ Surgical treatments, which increase bladder capacity and compliance using the large intestine or other organs, can be applied with limitations, due to the possible risk of surgical complications and/or mucous production in the augmented bladder. ${ }^{13}$ The frequent systemic side effects of medications, and short- and long-term complications of surgical interventions have prompted urologists to seek new treatment modalities.

Electrical stimulation of pudendal nerve afferents has advantages with its local effect, resulting in systemic side effects being lower than those caused by medication. This approach has been introduced as another effective treatment option for suppressing involuntary detrusor contractions in patients with NDO. Previous PNS studies have indicated that electrical stimulation of the pudendal nerve can inhibit the NVC during bladder filling, suppress reflex voiding, and increase MCC in animals and humans with chronic SCI, ${ }^{5,6}$ but there is no report regarding the inhibitory effect of PNS on bladder overactivity at the early stage of SCI. Our prior study shows that PNS can inhibit NVC during bladder filling in dogs with chronic SCI, but this can only increase MCC in the early period ( 1 month later). In the present study, group 1 underwent electrical stimulation of the pudendal nerve 1 day after transection (early stage). Stimulation was then applied 5 days a week ( $30 \mathrm{~min}$ each day) for 3 months at a

Table 1 Capacity and compliance of all dogs (mean \pm s.d.)

\begin{tabular}{|c|c|c|c|c|c|c|}
\hline & \multicolumn{3}{|c|}{ Capacity (ml) } & \multicolumn{3}{|c|}{ Compliance $\left(\mathrm{m} / \mathrm{cm}^{-1} \mathrm{H}_{2} \mathrm{O}\right)$} \\
\hline & Normal & 1 Month & 3 Months & Normal & 1 Month & 3 Months \\
\hline \multicolumn{7}{|c|}{ Group 1} \\
\hline 1 & $114.33 \pm 6.03$ & $110.00 \pm 10.00$ & $117.33 \pm 6.66$ & $7.99 \pm 1.83$ & $6.91 \pm 0.63$ & $8.23 \pm 0.80$ \\
\hline 2 & $113.00 \pm 3.00$ & $106.00 \pm 17.69$ & $126.00 \pm 5.29$ & $12.60 \pm 0.62$ & $9.77 \pm 0.40$ & $4.13 \pm 1.86$ \\
\hline 3 & $92.67 \pm 9.29$ & $85.33 \pm 5.03$ & $104.00 \pm 8.19$ & $4.85 \pm 0.43$ & $5.94 \pm 0.50$ & $5.84 \pm 0.23$ \\
\hline \multicolumn{7}{|c|}{ Group 2} \\
\hline 1 & $92.33 \pm 8.02$ & $15.67 \pm 4.16$ & $34.33 \pm 4.04$ & $4.20 \pm 0.03$ & $0.62 \pm 0.15$ & $0.77 \pm 0.06$ \\
\hline 2 & $117.33 \pm 9.29$ & $23.00 \pm 3.00$ & $47.00 \pm 2.65$ & $7.63 \pm 1.85$ & $1.57 \pm 0.21$ & $1.10 \pm 0.10$ \\
\hline 3 & $76.33 \pm 9.07$ & $31.67 \pm 7.77$ & $59.33 \pm 4.04$ & $5.74 \pm 0.77$ & $1.75 \pm 0.34$ & $1.38 \pm 0.25$ \\
\hline
\end{tabular}

Abbreviations: Normal, before spinal cord transection; 1 month, 1 month post-spinal cord transection; 3 months, 3 months post-spinal cord transection.
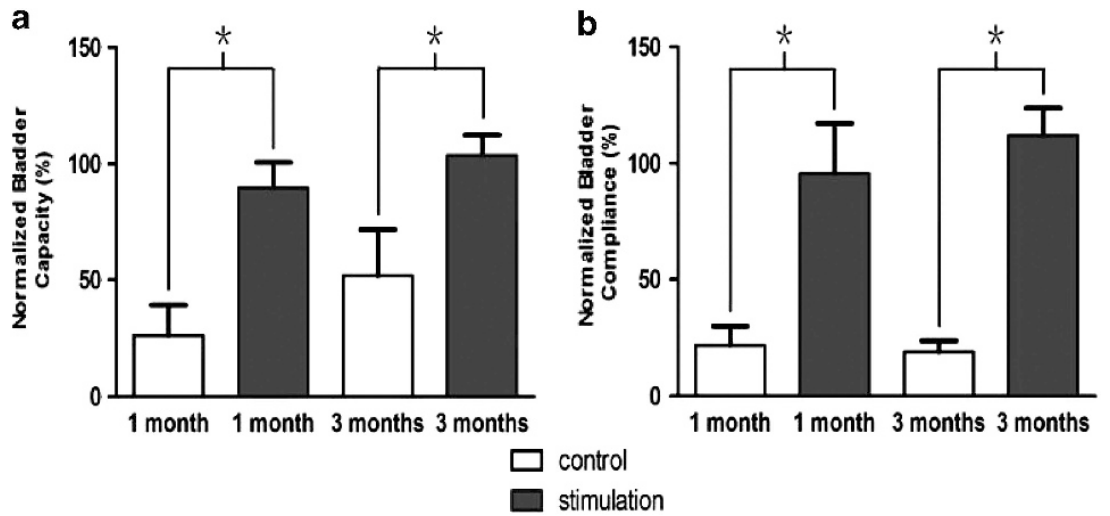

Figure 2 Bladder capacity (a) and compliance (b) were significantly increased $(P<0.05)$ in group 1 (early PNS) when compared with group 2 (no PNS) at 1 and 3 months post SCl. Asterick indicates statistical significance $(P<0.05)$. PNS, pudendal nerve stimulation. 

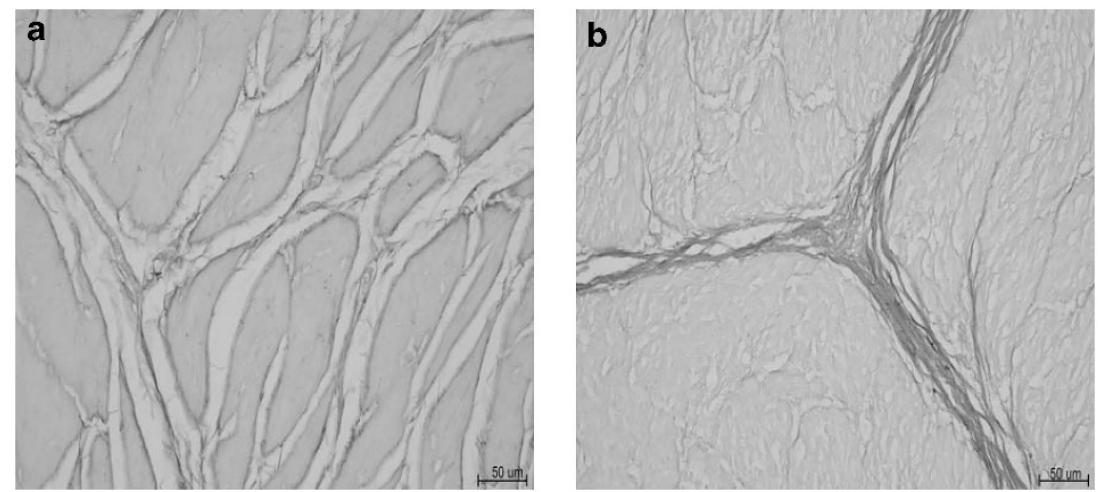

Figure 3 Ponceau S/Victoria blue staining. (a, b): Collagen (red) is significantly increased and elastin (blue) is significantly decreased in (b) (no PNS), compared with (a) (early PNS). Magnification, $\times 40$. PNS, pudendal nerve stimulation.

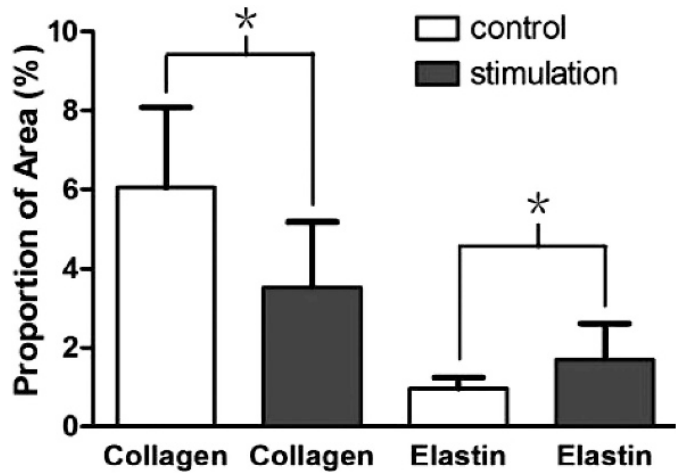

Figure 4 Image analysis shows that the proportion of collagen fibers is significantly increased and elastin fibers are significantly decreased in group 2 (no PNS) compared with group 1 (early PNS). Asterick indicates statistical significance $(P<0.05)$. PNS, pudendal nerve stimulation.

Table 2 Proportional area of collagen and elastin fibers (mean \pm s.d.)

\begin{tabular}{lccc}
\hline & Group 1 & Group 2 & P-value \\
\hline Collagen fibers (\%) & $3.53 \pm 1.65$ & $6.05 \pm 2.03$ & $<0.01$ \\
Elastin fibers (\%) & $1.70 \pm 0.92$ & $0.96 \pm 0.29$ & $<0.01$ \\
\hline
\end{tabular}

frequency of $5 \mathrm{~Hz}$. Group 2 did not undergo electrical stimulation of the pudendal nerve. We found that bladder capacity and compliance were significantly increased $(P<0.05)$ by early low-frequency pudendal nerve stimulation in group 1 when compared with group 2 at 1 and 3 months after SCI.

The exact neural mechanisms responsible for the effects of electrical neuromodulation on lower urinary tract dysfunction are unknown. A significant amount of research has focused on the effect of neuromodulation on afferent sensory nerve fibers, with the dominant theory being that electrical stimulation of these somatic afferent fibers modulates continence reflex pathways in the central nervous system. ${ }^{14}$ Acute SCI initially leads to detrusor acontractility and complete urinary retention, which is followed by slow overactivity development and urinary incontinence caused by C-fiber-mediated spinal reflex pathways. ${ }^{15}$ Early PNS during the bladder-areflexia phase might preserve nerve plasticity, such that C-fibers remain silent and detrusor overactivity is avoided. A previous study has found that low-frequency pudendal-afferent stimulation can evoke a robust reflex activation of hypogastric efferents. ${ }^{16}$ After SCI, hypogastric nerves may be intact. As such, low-frequency pudendal-afferent stimulation suppresses bladder contractions that may arise from activation of hypogastric efferent neurons, and subsequent synaptic and ganglionic inhibition of the parasympathetic-efferent neurons.

In patients with chronic SCI, their bladders become hypertrophic and/or fibrotic. ${ }^{17}$ It has been demonstrated that collagen synthesis and accumulation are increased during bladder fibrosis, and elastin levels are decreased. In this study, we found that collagen fibers were significantly increased, whereas elastin fibers were significantly decreased $(P<0.05)$ in group 2 (no PNS) when compared with group 1 (PNS). This suggested that low-frequency stimulation of the pudendal nerve at an early stage of SCI in dogs can delay the progress of bladder fibrosis.

Previous studies have proposed three possible mechanisms for the occurrence of bladder fibrosis after SCI. The first suggests that prolonged infection and stimulation cause structural changes in the bladder wall, such as fibrosis, which changes the passive properties of the detrusor muscle. ${ }^{18,19}$ The second possible mechanism is of myogenic origin, in which the denervated muscles change with the increase in collagen fibers and hypertrophy of smooth muscle fibers. ${ }^{20,21}$ The third possible mechanism is of neurogenic origin. ${ }^{22}$ In this study, we gave the group 1 dogs PNS at the early stage of SCI, and this might have imitated the effects of central nervous system and preserved nerve plasticity that would have been lost after complete SCI. Furthermore, low-frequency PNS may avoid the hypertrophy of bladder smooth muscle fibers resulting from suppressed bladder contractions. As such, early PNS may be an effective method for delaying the progress of bladder fibrosis following SCI, and may increase $\mathrm{BC}$, avoid vesicoureteral reflux and protect upper urinary tract function.

One potential criticism of this study is our method of BC calculation and the resulting potential bias. As there is no widely agreed-upon recommendations for calculating $\mathrm{BC}$ in animals, we calculated $\mathrm{BC}$ in our experimental dogs during the bladder-filling phase, according to the recommendation of the International Continence Society. ${ }^{8}$

Although we found that low-frequency PNS at the early stage of SCI could suppress bladder contractions and delay the progress of bladder fibrosis, further investigation will be necessary to determine the long-term outcomes, and whether stimulation has any other drawbacks or effects. In our future studies, we will perform ultrastructural study of the neurogenic bladder following treatment 
of early PNS to determine the changes in bladder smooth muscle cells and neuromuscular junctions to provide an empirical basis for the clinical application of early PNS.

\section{CONCLUSION}

This study shows that low-frequency PNS at the early stage of SCI can inhibit detrusor overactivity, increase bladder capacity and delay the progress of bladder fibrosis.

\section{DATA ARCHIVING}

There were no data to deposit.

\section{CONFLICT OF INTEREST}

The authors declare no conflict of interest.

\section{ACKNOWLEDGEMENTS}

The study was supported by the National Natural Science Foundation of China (Grant no. 81070607) and the China National Key Technology R\&D Program (no. 2012BAI34B02).

1 Benevento BT, Sipski ML. Neurogenic bladder, neurogenic bowel, and sexual dysfunction in people with spinal cord injury. Phys Ther 2002; 82: 601-612.

2 Burns AS, Rivas DA, Ditunno JF. The management of neurogenic bladder and sexual dysfunction after spinal cord injury. Spine 2001; 26: 129-136.

3 Riccabona M, Koen M, Schindler M, Goedele B, Pycha A, Lusuardi L et al. BotulinumA toxin injection into the detrusor: a safe alternative in the treatment of children with myelomeningocele with detrusor hyperreflexia. J Urol 2004; 171: 845-848.

4 Shingleton WB, Bodner DR. The development of urologic complications in relationship to bladder pressure in spinal cord injured patients. J Am Paraplegia Soc 1993; 16: 14-17

5 Tai C, Wang J, Wang X, de Groat WC, Roppolo JR. Bladder inhibition or voiding induced by pudendal nerve stimulation in chronic spinal cord injured cats. Neurourol Urodyn 2007; 6: 570-577.

6 Lee YH, Kim JM, Im HT, Lee KW, Kim SH, Hur DM. Semiconditional electrical stimulation of pudendal nerve afferents stimulation to manage neurogenic detrusor overactivity in patients with spinal cord injury. Ann Rehabil Med 2011; 35: 605-612.

7 Opisso E, Borau A, Rodrigues A, Hansen J, Rijkhoff NJ. Patient controlled versus automatic stimulation of pudendal nerve affferents to treat neurogenic detrusor overactivity. J Urol 2008; 180: 1404-1408.

8 Chen G, Liao L, Dong Q, Ju Y. The Inhibitory effects of pudendal nerve stimulation on bladder overactivity of spinal cord injury in dogs: is early stimulation necessary? Neuromodulation 2012; 15: 232-237.

9 Abrams P, Cardozo L, Fall M, Griffiths D, Rosier P, Ulmsten U. The standardisation of terminology of lower urinary tract function: Report from the Standardisation Sub-committee of the International Continence Society. Neurourol Urodyn 2002; 21: 167-168.

10 Schäfer W, Abrams P, Liao L, Mattiasson A, Pesce F, Spangberg A. Good urodynamic practices: uroflowmetry, filling cystometry, and pressure-flow studies. Neurourol Urodyn 2002; 21: 261-274.

11 Perkash I. Long-term urologic management of the patient with spinal cord injury. Urol Clin North Am 1993; 20: 423-434.

12 Sahai A, Khan MS, Gregson N, Smith K, Dasgupta P, GKT Botulinum Study Group. Botulinum toxin for detrusor overactivity and symptoms of overactive bladder: where we are now and where we are going. Nat Clin Pract Urol 2007; 4: 379-386.

13 Changfeng T, Werner S, Warren MG, Karl-Dietrich S, Juan W, Yanhe J. Treatment of overactive bladder. ISS 2004; 7: 163-174.

14 Leng WW. Chancellor MB. How sacral nerve stimulation neuromodulation works. Urol Clin North Am 2005; 23: 11-18.

15 De Groat WC, Yoshimura N. Mechanisms underlying the recovery of lower urinary tract function following spinal cord injury. Prog Brain Res 2006; 152 59-84.

16 Lindström S, Fall M, Carlsson CA, Erlandson BE. The neurophysiological basis of bladder inhibition in response to intravaginal electrical stimulation. J Urol 1983; 129: 405-410.

17 Deveaud CM, Macarak EJ, Kucich U, Ewalt DH, Abrams WR, Howard PS. Molecular analysis of collagens in bladder fibrosis. J Urol 1998; 160: 1518-1527.

18 German K, Bedwani J, Davies J, Brading AF, Stephenson TP. An assessment of the contribution of visco-elastic factors in the aetiology of poor compliance in the human neuropathic bladder. Br J Urol 1994; 74: 744-748.

19 McGuire EJ, Morrissey SG. The development of neurogenic vesical dysfunction after experimental spinal cord injury or sacral rhizotomy in non-human primates. J Urol 1982; 128: 1390-1393.

20 Ghoniem GM. Effect of bilateral sacral decentralization on detrusor contractility and passive properties in dogs. Neurourol Urodyn 1984; 3: 23-30.

21 Brading AF, Turner $\mathrm{WH}$. The unstable bladder: towards a common mechanism. Br J Urol 1994; 73: 3-8.

22 Uvelius B, Mattiasson A. Detrusor collagen content in the denervated rat bladder J Urol 1986; 136: 601-604. 